\title{
Relationship between variations in posterior vitreous detachment and visual prognosis in idiopathic epiretinal membranes
}

This article was published in the following Dove Press journal:

Clinical Ophthalmology

2I December 2015

Number of times this article has been viewed

\section{Ayumi Ota \\ Yoshiaki Tanaka \\ Fumihiko Toyoda \\ Machiko Shimmura \\ Nozomi Kinoshita \\ Hiroko Takano \\ Akihiro Kakehashi}

Department of Ophthalmology, Jichi Medical University, Saitama Medical

Center, Omiya-ku, Saitama, Japan
Correspondence: Akihiro Kakehashi Department of Ophthalmology, Jichi Medical University, Saitama Medical Center, I-847 Amanuma-cho, Omiya-ku, Saitama 330-8503, Japan Email kakeaki@omiya.jichi.ac.jp
Purpose: To clarify the relationship between variations in posterior vitreous detachments (PVDs) and visual prognoses in idiopathic epiretinal membranes (ERMs).

Methods: In this retrospective, observational, and consecutive case series, we observed variations in PVDs in 37 patients (mean age, 65.7 \pm 11.0 years) with ERMs and followed them for 2 years. Three PVD types were found biomicroscopically: no PVD, complete PVD with collapse (C-PVD with collapse), and partial PVD without shrinkage, with persistent vitreous attachment to the macula through the premacular hole of the posterior hyaloid membrane (P-PVD without shrinkage [M]). The best-corrected visual acuity (BCVA) was measured and converted to the logarithm of the minimum angle of resolution (logMAR) BCVA at the first visit and 2 years later.

Results: No PVD was observed in 16 of the 37 eyes (mean age, $61.3 \pm 11.3$ years), C-PVD with collapse in 11 of the 37 eyes (mean age, 69.1 99.9 years), and P-PVD without shrinkage (M) in 10 of the 37 eyes (mean age, $69.3 \pm 10.9$ years). The logMAR BCVA at the first visit was the worst in the P-PVD without shrinkage (M) group (0.22 \pm 0.35$)$ compared with the no-PVD group $(-0.019 \pm 0.07 ; P<0.01)$ and the C-PVD group $(0.029 \pm 0.08 ; P<0.05)$. The logMAR BCVA 2 years later was also worst in the P-PVD without shrinkage $(\mathrm{M})$ group $(0.39 \pm 0.35)$ compared with the no-PVD group $(0.04 \pm 0.13)$ and the C-PVD with collapse group $(0.03 \pm 0.09 ; P<0.05$ for both comparisons). The change in the logMAR BCVA over the 2-year follow-up period was worst in the P-PVD without shrinkage $(\mathrm{M})$ group $(0.17 \pm 0.23)$ compared with the no-PVD group $(0.06 \pm 0.14)$ and the C-PVD with collapse group ( $0.0009 \pm 0.09 ; P<0.05$ for both comparisons).

Conclusion: Cases with an ERM with a P-PVD without shrinkage (M) had a worse visual prognosis than those with an ERM with no PVD and C-PVD with collapse.

Keywords: posterior vitreous detachment, idiopathic epiretinal membranes, visual prognosis

\section{Introduction}

Epiretinal membrane (ERM) is the most common retinal disease in adults. ${ }^{1-3}$ ERMs occur spontaneously or secondarily almost always at the macular area and contain several cellular types, ie, fibrous astrocytes, retinal pigment epithelial cells, fibrocytes, and macrophages, ${ }^{4}$ as well as substantial collagen. ${ }^{5}$ Shrinkage of the ERM causes macular pucker and subsequent symptoms of visual loss and metamorphopsia. The symptoms of the ERM vary from none to severe. Generally, there are minimal symptoms of visual loss and metamorphopsia. In the natural history, the progression of pathology and symptoms of ERMs is usually slow. Sidd et al reported that $90 \%$ of eyes with an idiopathic ERM maintained the same level of visual acuity (VA) during a 31-month follow-up period. ${ }^{1}$ However, because certain patients with an idiopathic ERM reported visual deterioration, the ERM must be removed to prevent further 
deterioration and recover the vision. However, the effect of vitrectomy is unsatisfactory for advanced cases of ERMs. ${ }^{6,7}$ Early vitrectomy should be indicated in certain cases with idiopathic ERMs. Thus, we need to determine both what types of ERMs progress rapidly among the idiopathic ERMs and the candidates for early vitrectomy.

Many reports have clarified that posterior vitreous detachments (PVDs) are strongly associated with the formation of ERMs. ${ }^{1,8-10}$ Although some reports have mentioned that the prognosis is based primarily on the findings of optical coherence tomography (OCT), ${ }^{11-13}$ few reports have mentioned an association between the PVD type and the prognoses of ERMs. ${ }^{9}$ We previously presented several cases of the macular disease in which the visual prognosis was considered to be affected by a certain type of partial PVD. ${ }^{14}$

The purpose of the current study was to clarify the relationship between the PVD type and visual prognoses in idiopathic ERMs and identify the candidates for early vitrectomy among them.

\section{Methods}

We observed variations in PVDs by both biomicroscopy and OCT in the eyes of 37 patients (mean age, $65.7 \pm 11.0$ years) with idiopathic ERMs and followed the patients for 2 years. The PVD variations were classified biomicroscopically according to the PVD classification of Kakehashi et al (Figure 1). ${ }^{14,15}$ The vitreous conditions initially were divided into no-PVD and PVD. The PVDs were then classified as complete PVDs (C-PVDs) and partial PVDs (P-PVDs). The C-PVDs were divided into C-PVDs with collapse and without collapse, and the P-PVDs into P-PVD with shrinkage of the posterior hyaloid membrane (P-PVDs with shrinkage) and without shrinkage of the posterior hyaloid membrane (P-PVDs without shrinkage). The P-PVDs without shrinkage have a subtype with vitreous gel attachment through the premacular hole in the posterior hyaloid membrane to the macula (P-PVDs without shrinkage $[\mathrm{M}]$ ). Biomicroscopy results showed that ERM cases with P-PVDs without shrinkage (M) had a persistent vitreous attachment to the macula through the premacular hole of the posterior hyaloid membrane (Video S1) but no apparent vitreous traction on OCT images (Figure 2).

The cases of the classic vitreomacular traction syndrome were classified as a P-PVD with shrinkage (Figure 3), which were excluded from the current study, because the visual prognosis of ERM cases with vitreomacular traction syndrome is not good and requires vitreous surgery. ${ }^{7,16,17} \mathrm{We}$ need to determine the visual prognosis of the other types of idiopathic ERMs.

The best-corrected VA (BCVA) was measured several times during the 2-year follow-up period. The BCVA was

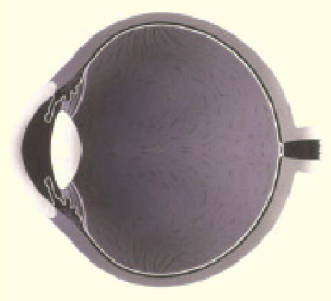

No PVD

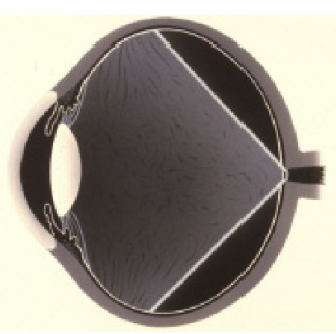

P-PVD with shrinkage

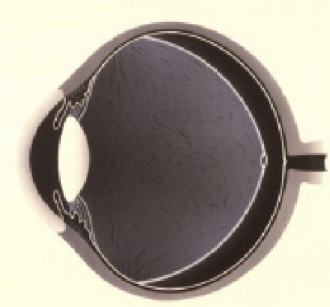

C-PVD without collapse

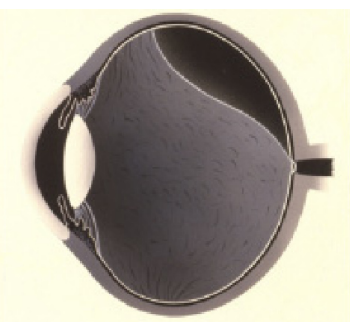

P-PVD without shrinkage

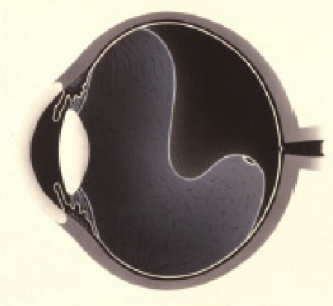

C-PVD with collapse

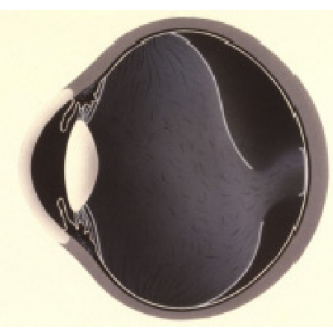

P-PVD without shrinkage (M)

Figure I Classification of posterior vitreous detachments (PVDs) by slit-lamp biomicroscopy

Abbreviations: No PVD, no posterior vitreous detachment; C-PVD without collapse, complete posterior vitreous detachment without collapse; C-PVD with collapse, complete posterior vitreous detachment with collapse; P-PVD with shrinkage, partial posterior vitreous detachment with shrinkage of the posterior hyaloid membrane; P-PVD without shrinkage, partial posterior vitreous detachment without shrinkage of the posterior hyaloid membrane; P-PVD without shrinkage (M), P-PVD without shrinkage showing vitreous gel attachment to the macula through the premacular hole in the posterior hyaloid membrane. 


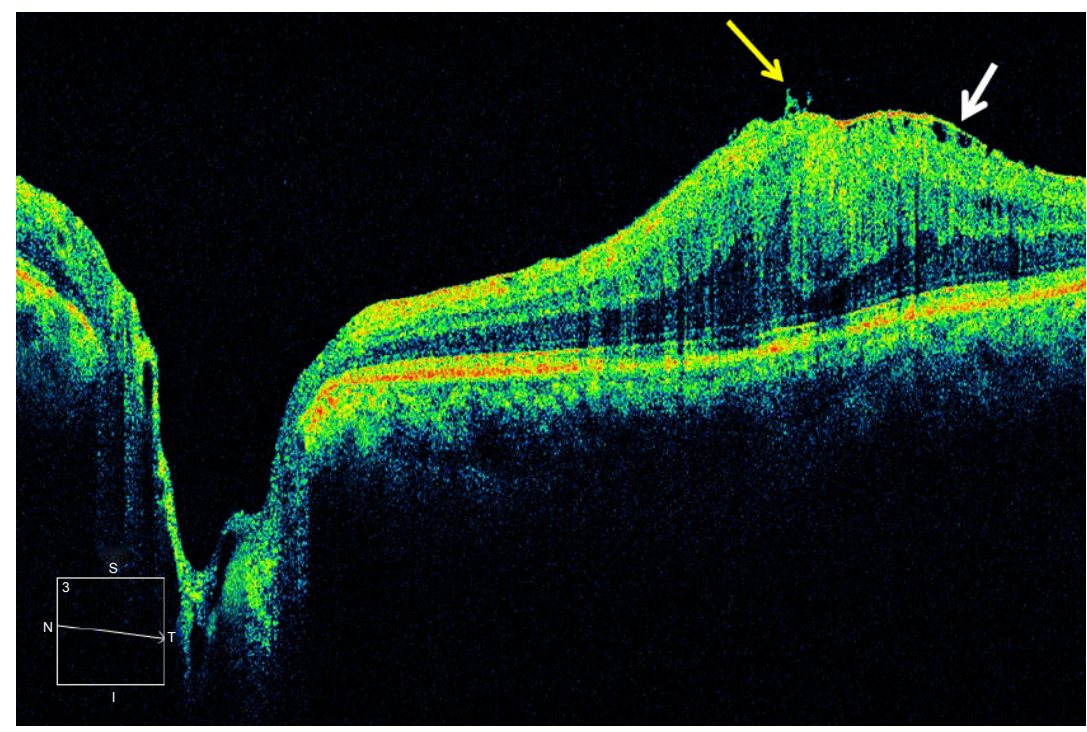

Figure 2 OCT image of an ERM case with P-PVDs without shrinkage (M).

Notes: An epiretinal membrane (white arrow) is seen clearly by optical coherence tomography, but vitreous gel traction on the macula is hardly detected except for just in front of the macula (yellow arrow). However, biomicroscopy shows chronic vitreous traction on the macula as a posterior vitreous detachment (PVD) type of partial PVD without shrinkage (M). The white box shows scan direction form nasal to temporal.

Abbreviations: $\mathrm{N}$, nasal; $\mathrm{S}$, superior; $\mathrm{T}$, temporal; I, inferior.

converted to the logarithm of the minimum angle of resolution (logMAR) units, and the BCVA at the first visit and 2 years later were compared in each case. The cases with corneal opacity, cataract, and vitreous opacity that interfered with biomicroscopy and OCT were excluded. The cases with other retinal diseases, such as diabetic retinopathy, hypertensive retinopathy, retinal vein occlusion, and high myopia and those with a history of uveitis and ocular surgery, were also excluded.

All values were expressed as mean \pm standard deviation. The Mann-Whitney $U$-test and Steel's test were used for comparisons between each group. Excel Tokei 2006 software (Social Survey Research Information Co., Ltd., Tokyo, Japan) was used for statistical analyses. $P<0.05$ was considered

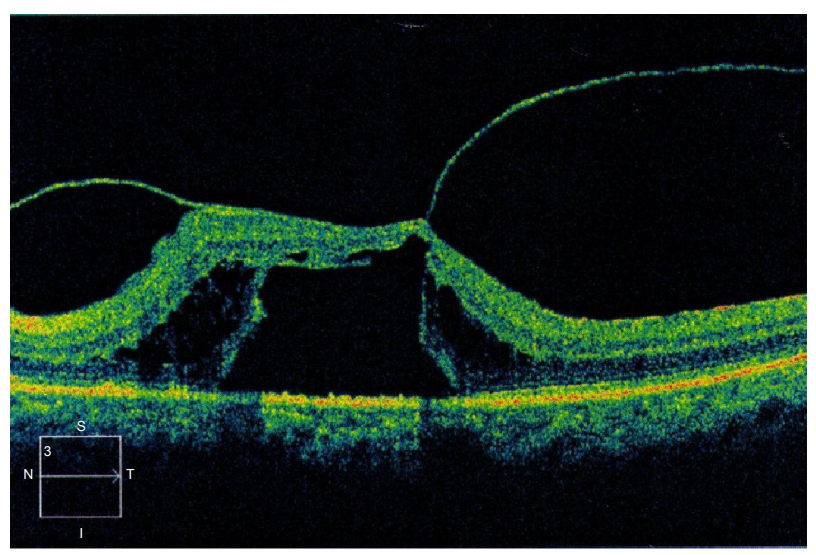

Figure $3 \mathrm{~A}$ case of classic vitreomacular traction syndrome classified as partial posterior vitreous detachment with shrinkage.

Note: A table top-type tractional retinal detachment is seen clearly by optical coherence tomography.

Abbreviations: $\mathrm{N}$, nasal; S, superior; T, temporal; I, inferior. statistically significant. The study protocol was approved by ethics committees of our institution, and informed consent was obtained from all participants. This research adhered to the tenets of the Declaration of Helsinki.

\section{Results}

We observed the following three types of PVDs: no-PVD, C-PVD with collapse, and P-PVD without shrinkage (M) in this study. No case had a C-PVD without collapse. All cases with a P-PVD without shrinkage were categorized as a P-PVD without shrinkage (M).

At the first visit, no PVD was observed in 16 of the 37 eyes (mean age, $61.3 \pm 11.3$ years), a C-PVD with collapse in 11 of the 37 eyes (mean age, 69.1 \pm 9.9 years), and a P-PVD without shrinkage (M) in 10 of the 37 eyes (mean age, $69.3 \pm 10.9$ years). Each PVD type did not change over the 2-year follow-up period.

At the first visit, the logMAR BCVA was the worst in the P-PVD without shrinkage (M) group $(0.22 \pm 0.35)$ compared with the no-PVD group $(-0.019 \pm 0.07 ; P<0.01)$ and the C-PVD with collapse group $(0.029 \pm 0.08 ; P<0.05)$. Two years later, the $\log M A R$ BCVA remained the worst in the P-PVD without shrinkage (M) group (0.39 \pm 0.35$)$ compared with the no-PVD group $(0.04 \pm 0.13)$ and the C-PVD group with collapse $(0.03 \pm 0.09 ; P<0.05$ for both comparisons). The $\operatorname{logMAR}$ BCVA in the P-PVD group without shrinkage (M) $(0.17 \pm 0.23)$ deteriorated significantly $(P<0.05)$ during the 2 -year follow-up period. However, the logMAR BCVA did not deteriorate significantly in the no-PVD group $(0.06 \pm 0.14)$ 


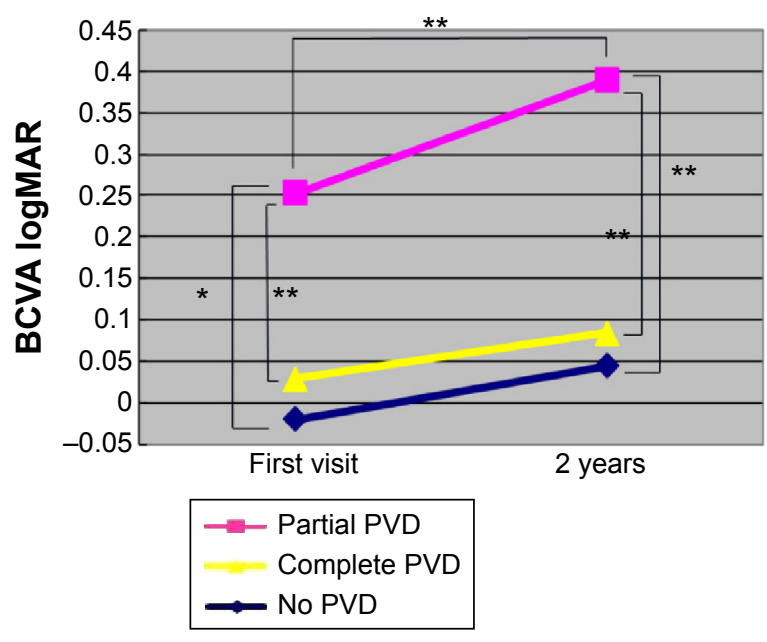

Figure 4 The visual prognosis in each group.

Notes: The logarithm of the minimum angle of resolution of the best-corrected visual acuity (logMAR BCVA) is worst in the P-PVD without shrinkage (M) group compared with the no-PVD group and the C-PVD with collapse group. The logMAR BCVA 2 years later is also worst in the P-PVD without shrinkage $(M)$ group compared with the no-PVD group and the C-PVD group. The logMAR BCVA has deteriorated significantly during the 2-year follow-up period in the P-PVD group. However, in the no-PVD group and the C-PVD with collapse group, the logMAR BCVA has not deteriorated significantly during the 2 -year follow-up. $* P<0.05, * * P<0.01$.

Abbreviations: PVD, posterior vitreous detachment; P-PVD, partial posterior vitreous detachment; C-PVD, complete posterior vitreous detachment.

and the C-PVD with collapse group $(0.0009 \pm 0.09)$ during the 2-year follow-up (Figure 4).

\section{Discussion}

Among all types of idiopathic ERMs, the visual prognosis is the worst in the idiopathic ERM cases complicated by the classic vitreomacular traction syndrome..$^{7,16,17}$ Both tangential vitreous traction and anteroposterior vitreous traction are exerted on the macula in those cases. Therefore, in those cases with classic vitreomacular traction syndrome, the recommendation is to remove the ERM to maintain or recover vision. It is easy to diagnose idiopathic ERMs with classic vitreomacular traction syndrome on OCT images.

In the current study, we excluded cases with the classic vitreomacular traction syndrome and then identified three types of PVDs in ERM cases: no PVD, C-PVD with collapse, and P-PVD without shrinkage (M). In these cases, we did not find anteroposterior vitreous traction on the OCT images. We observed tangential traction on the macula on OCT images but did not observe anteroposterior vitreous traction alone in these cases. This may be due to the resolution limitation of SD-OCT. The vitreous is mobile and most cases have few extracellular matrices and a large amount of water. Meticulous vitreous biomicroscopy is needed to evaluate weak and chronic anteroposterior vitreous traction on the macular (Video S1).

$\mathrm{Jaffe}^{18}$ initially reported this type of vitreous pathology, and Sebag and Balazs later confirmed it by in vitro slit-lamp microscopy. ${ }^{19} \mathrm{We}$ also confirmed this vitreous pathology in vivo by scanning laser ophthalmoscopy (Video S2) ${ }^{20}$ In the current study, we found that idiopathic ERMs with a P-PVD without shrinkage (M) had the worst visual prognosis compared with the other types of ERMs, possibly because of the chronic weak vitreous traction exerted on the macula. We also theorized that certain cells, such as macrophages or pigment epithelial cells, can migrate via the vitreous to the macula. Cracking of the internal limiting membrane can also occur as the result of chronic vitreous traction, facilitating the migration of glial cells from the retina to the macula via the crack. We should consider removing ERMs in their early stage in cases of ERMs with a P-PVD without shrinkage (M).

Other types of ERMs can be treated without surgery because the vision tends to remain unchanged for a long time. In the current study, ERMs with no PVD and C-PVD with collapse were associated with minimal deterioration of the logMAR BCVA for over 2 years. An examination of the vitreoretinal interface and retinal layer, especially the photoreceptor inner/outer segment junction line and cone outer segment tip line, using OCT is important for determining the diagnosis, natural course, and surgical prognosis of the ERMs. ${ }^{7,1321-23}$ In addition to these OCT examinations, basic slit-lamp vitreous biomicroscopy is also important for this purpose, particularly in the early stage.

Even though the results were statistically significant, a large sample size and a long and relatively frequent observation period are necessary in future investigations to provide more powerful evidence. Since vitrectomy is associated with complications, the candidates for early surgery should be carefully identified.

\section{Conclusion}

ERMs with a P-PVD without shrinkage (M) had the worst visual prognosis compared with ERMs with no PVD and a C-PVD with collapse. Chronic vitreous traction on the ERM may cause the poor visual prognosis associated with ERMs. Correct diagnosis of the type of PVD by OCT and vitreous biomicroscopy is important not only to evaluate the visual prognosis but also to determine the need for surgery to remove idiopathic ERMs.

\section{Author contributions}

The design and conduct of the study was performed by AO and AK. Acquisition and management of data were carried 
out by AO, YT, FT, MS, NK, HT, and AK. Analysis and interpretation of the data were carried out by $\mathrm{AO}$ and $\mathrm{AK}$. All authors contributed toward drafting and critically revising the paper and agree to be accountable for all aspects of the work.

\section{Disclosure}

The authors report no conflicts of interest in this work.

\section{References}

1. Sidd RJ, Fine SL, Owens SL, Patz A. Idiopathic preretinal gliosis. Am J Ophthalmol. 1982;94(1):44-48.

2. Klein R, Klein BE, Wang Q, Moss SE. The epidemiology of epiretinal membranes. Trans Am Ophthalmol Soc. 1994;92:403-425. [discussion 425-430].

3. Mitchell P, Smith W, Chey T, Wang JJ, Chang A. Prevalence and associations of epiretinal membranes. The Blue Mountains Eye Study, Australia. Ophthalmology. 1997(6);104:1033-1040.

4. Kampik A, Kenyon KR, Michels RG, Green WR, de la Cruz ZC. Epiretinal and vitreous membranes. Comparative study of 56 cases. Arch Ophthalmol. 1981;99(8):1445-1454.

5. Trese M, Chandler DB, Machemer R. Macular pucker. II. Ultrastructure. Graefe's Arch Clin Exp Ophthalmol. 1983;221(1):16-26.

6. Roe RH, McDonald HR, Fu AD, et al. Unexplained vision loss following removal of epiretinal membrane. Br J Ophthalmol. 2010;94(8): 1033-1039.

7. Kinoshita T, Kovacs KD, Wagley S, Arroyo JG. Morphologic differences in epiretinal membranes on ocular coherence tomography as a predictive factor for surgical outcome. Retina. 2011;31(8):1692-1698.

8. Wise GN. Relationship of idiopathic preretinal macular fibrosis to posterior vitreous detachment. Am J Ophthalmol. 1975;79(3):358-362.

9. Hirokawa H, Jalkh AE, Takahashi M, Trempe CL, Schepens CL. Role of the vitreous in idiopathic preretinal macular fibrosis. Am J Ophthalmol. 1986; 101(2):166-169.

10. Suh MH, Seo JM, Park KH, Yu HG. Associations between macular findings by optical coherence tomography and visual outcomes after epiretinal membrane removal. Am J Ophthalmol. 2009;147(3):473-480
11. Falkner-Radler CI, Glittenberg C, Hagen S, Benesch T, Binder S. Spectral-domain optical coherence tomography for monitoring epiretinal membrane surgery. Ophthalmology. 2010;117(4):798-805.

12. Kim JH, Kim YM, Chung EJ, Lee SY, Koh HJ. Structural and functional predictors of visual outcome of epiretinal membrane surgery. $\mathrm{Am}$ J Ophthalmol. 2012;153(1):103-110.

13. Shimozono M, Oishi A, Hata M, et al. The significance of cone outer segment tips as a prognostic factor in epiretinal membrane surgery. $\mathrm{Am}$ J Ophthalmol. 2012;153:698-704.

14. Kakehashi A, Takezawa M, Akiba J. Classification of posterior vitreous detachment. Clin Ophthalmol. 2014;8:1-10.

15. Kakehashi A, Kado M, Akiba J, Hirokawa H. Variations of posterior vitreous detachment. Br J Ophthalmol. 1997;81(7):527-532.

16. Chang LK, Fine HF, Spaide RF, Koizumi H, Grossniklaus HE. Ultrastructural correlation of spectral-domain optical coherence tomographic findings in vitreomacular traction syndrome. Am J Ophthalmol. 2008;146(1): $121-127$.

17. McDonald HR, Johnson RN, Schatz H. Surgical results in the vitreomacular traction syndrome. Ophthalmology. 1994;101(8):1397-1402.

18. Jaffe NS. Vitreous traction at the posterior pole of the fundus due to alterations in the vitreous posterior. Trans Am Acad Ophthalmol Otolaryngol. 1967;71(4):642-652.

19. Sebag J, Balazs EA. Morphology and ultrastructure of human vitreous fibers. Invest Ophthalmol Vis Sci. 1989;30(8):1867-1871.

20. Kakehashi A, Ishiko S, Konno S, Akiba J, Kado M, Yoshida A. Observing the posterior vitreous by means of the scanning laser ophthalmoscope. Arch Ophthalmol. 1995;113(5):558-560.

21. Johnson MW. Posterior vitreous detachment: evolution and complications of its early stages. Am J Ophthalmol. 2010;149(3):371-382

22. Watanabe K, Tsunoda K, Mizuno Y, Akiyama K, Noda T. Outer retinal morphology and visual function in patients with idiopathic epiretinal membrane. JAMA Ophthalmol. 2013;131(2):172-177.

23. Itoh $Y$, Inoue M, Rii T, Hirota K, Hirakata A. Correlation between foveal cone outer segment tips line and visual recovery after epiretinal membrane surgery. Invest Ophthalmol Vis Sci. 2013;54(12):7302-7308.
Clinical Ophthalmology

\section{Publish your work in this journal}

Clinical Ophthalmology is an international, peer-reviewed journa covering all subspecialties within ophthalmology. Key topics include: Optometry; Visual science; Pharmacology and drug therapy in eye diseases; Basic Sciences; Primary and Secondary eye care; Patient Safety and Quality of Care Improvements. This journal is indexed on

\section{Dovepress}

PubMed Central and CAS, and is the official journal of The Society of Clinical Ophthalmology (SCO). The manuscript management system is completely online and includes a very quick and fair peer-review system, which is all easy to use. Visit http://www.dovepress.com/ testimonials.php to read real quotes from published authors. 